\title{
Why use Ionic Liquids for Electrodeposition?
}

Andrew P. Abbott, Ian Dalrymple, Frank Endres, and Douglas R. MacFarlane

With any great voyage of discovery the explorer should always be asked at the outset "Why are you doing this?" To answer the question "Why use ionic liquids for electrodeposition?" it is first necessary to look at current best practice and find its limitations.

It is widely recognised that in 1805 Italian chemist, Luigi Brugnatelli made the first experiments in what we now know as electroplating. Brugnatelli used the newly discovered Voltaic Pile to deposit gold "I have lately gilt in a complete manner two large silver medals, by bringing them into communication by means of a steel wire, with a negative pole of a voltaic pile, and keeping them one after the other immersed in ammoniuret of gold newly made and well saturated" [1]. The process was later improved by John Wright who found that potassium cyanide was a beneficial electrolyte to add for silver and gold plating as it allowed thick adherent deposits to be obtained. Until the middle of the 19th century the production of jewellery and the gilding of decorative items were the main uses of electrodeposition.

With an increased understanding of electrochemistry, the practice of metal deposition spread to non-decorative metals such as nickel, brass, tin, and zinc by the 1850s. Even though electroplated goods entered many aspects of manufacturing industry very little changed about the physical processes involved in electrodeposition for about 100 years. It was only with the advent of the electronics industry in the middle of the 20th century that significant changes occurred in the hardware and chemistry of the plating solutions. The post-war period saw an increase in gold plating for electronic components and the use of less hazardous plating solutions. This trend has continued with increased control of hazardous materials to the environment. Improved solution composition and power supply technology has allowed the development of fast and continuous plating of wire, metal strips, semiconductors and complex substrate geometries.

Many of the technological developments seen in the electronics industry depend upon sophisticated electroplating including the use of exotic metals and this is one of the drivers for new technology within the electroplating sector. The other

Electrodeposition from Ionic Liquids. Edited by F. Endres, D. MacFarlane, A. Abbott Copyright (C) 2008 WILEY-VCH Verlag GmbH \& Co. KGaA, Weinheim ISBN: 978-3-527-31565-9 
1 Why use Ionic Liquids for Electrodeposition?

main driver is the search for alternative technologies for metals such as chromium, nickel and cadmium. Anti-corrosion and wear-resistant coatings are predominant markets in the electroplating sector and environmental directives will evidently limit their usage in the future.

The main metals of that commercially deposited are $\mathrm{Cr}, \mathrm{Ni}, \mathrm{Cu}, \mathrm{Au}, \mathrm{Ag}, \mathrm{Zn}$ and $\mathrm{Cd}$ together with a number of copper and zinc-based alloys [1]. The whole electroplating sector is based on aqueous solutions. There are some niche markets based on organic solvents such as aluminum but these are very much exceptions. Metals outside this list are generally deposited using plasma or chemical vapor deposition techniques (PVD and CVD). These methods allow the coating of most substrates (metal, plastic, glass, ceramic etc.) not only with metal but also with alloys or compounds (oxide, nitride, carbide, etc.), without damaging the environment. Although these techniques are technically interesting, it is regrettable that they always involve high capital investment and it is difficult to prepare thick coatings, thus they are only applied to high value niche markets.

Clearly the key advantages of using aqueous solutions are:

- Cost

- Non-flammable

- High solubility of electrolytes

- High conductivities resulting in low ohmic losses and good throwing power

- High solubility of metal salts

- High rates of mass transfer.

For these reasons water will remain the mainstay of the metal plating industry, however, there are also limitations of aqueous solutions including:

- Limited potential windows

- Gas evolution processes can be technically difficult to handle and result in hydrogen embrittlement

- Passivation of metals can cause difficulties with both anodic and cathodic materials

- Necessity for complexing agents such as cyanide

- All water must eventually be returned to the water course.

These prevent aqueous solutions being applied to the deposition of several technically important materials.

The key technological goals include replacement of environmentally toxic metal coatings, deposition of new alloys and semiconductors and new coating methods for reactive metals. The main driving force for non-aqueous electrolytes has been the desire to deposit refractory metals such as $\mathrm{Ti}, \mathrm{Al}$ and $\mathrm{W}$. These metals are abundant and excellent for corrosion resistance. It is, however, the stability of their oxides that makes these metals difficult to extract from minerals and apply as surface coatings. 


\section{1}

\section{Non-aqueous Solutions}

There is clearly a range of alternative non-aqueous solutions that could be used. Ideally, to obtain the properties required for an electrolyte solution, polar molecules have to be used and these should preferably be small to obtain the requisite high fluidity. Unfortunately, all polar molecules result from having electronegative elements which by their nature makes them good electron donors. Accordingly, they will strongly coordinate to metal ions making them difficult to reduce. While a number of metals have been deposited from polar organic solvents these tend to be the rather noble metals and the processes offer few advantages over aqueous solutions. Some studies have been made using non-polar organic solvents, predominantly aromatic hydrocarbons but these suffer from the serious disadvantage that the dissolved electrolytes are highly associated and the solutions suffer from poor conductivity. The solutions do, however, have wide potential windows and it has been demonstrated that metals such as aluminum and titanium (Ti at least in very thin layers) can be deposited from them. One of the most successful non-aqueous processes is the SIGAL process developed in the late 1980s for the deposition of aluminum from toluene [2, 3]. The aluminum source is triethyl aluminum which is pyrophoric and, despite the high flammability of the electrolyte solution, the process has been commercialized and is currently the only electrochemical method for the deposition of aluminum. A review of electrochemistry in non-aqueous solutions is given by Izutsu [4].

\section{2}

\section{Ionic Fluids}

Clearly an alternative to molecular solvents is the use of ionic fluids. Ionic materials usually melt at high temperatures due to their large lattice energies. Hightemperature molten salts have been extensively used for the electrowinning of metals such as $\mathrm{Li}, \mathrm{Na}, \mathrm{Ti}$ and $\mathrm{Al}$ at temperatures of up to $1000^{\circ} \mathrm{C}$ [5-7]. They have wide potential windows, high conductivities and high solubilities for metal salts, in fact they have most of the advantages of aqueous solutions and overcome most of the limitations of aqueous solutions, but clearly they suffer from the major limitation that the operational conditions are difficult to achieve and limit the range of substrates that can be used for deposition.

The alternative to high-temperature molten salts is to use an ionic substance that melts at a low temperature. While this may sound like an oxymoron it is logical to suppose that the melting point of an ionic substance is related to ionic size and if the ions are made large enough the material will eventually melt at ambient conditions. A significant amount of work was carried out in the middle of the 20th century with the aim of developing lower temperature molten salts. One of the key aims was a lower temperature melt for aluminum deposition which led 
$4 \quad 1$ Why use lonic Liquids for Electrodeposition?

to the formation of $\mathrm{Li}^{+} / \mathrm{K}^{+} / \mathrm{AlCl}_{3}$ eutectics which have freezing points close to $100{ }^{\circ} \mathrm{C}[8]$. These low freezing points arise due to the large chloroaluminate anions $\left(\mathrm{AlCl}_{4}{ }^{-}\right.$and $\left.\mathrm{Al}_{2} \mathrm{Cl}_{7}{ }^{-}\right)$that form in the eutectic mixtures and have low lattice energies. The use of quaternary ammonium salts, particularly pyridinium and imidazolium salts, has pushed the freezing point down to ambient conditions. The term "ionic liquids" was coined to distinguish these lower temperature ionic fluids from the high temperature analogues which are composed predominantly of inorganic ions.

The synthesis and properties of a range of ionic liquids are briefly summarized in the following chapter while the history and chemical properties of these liquids are covered in several well known reviews [9-12]. Several applications of ionic liquids are being tested and these are as diverse as fuel desulfurization [13] and precious metal processing [14] but few have yet come to practical fruition.

BASF's BASIL process [15] and the Dimersol ${ }^{\circledR}$ process [16] have both been commercialized. The former uses the ionic liquid as a phase transfer catalyst to produce alkoxyphenylphosphines which are precursors for the synthesis of photoinitiators used in printing inks and wood coatings. The imidazole acts as a proton scavenger in the reaction of phenyl-chlorophosphines with alcohols to produce phosphines. The Dimersol ${ }^{\circledR}$ process uses a Lewis acid catalyst for the dimerization of butenes to produce $\mathrm{C}_{8}$ olefins which are usually further hydroformylated giving $\mathrm{C}_{9}$ alcohols used in the manufacture of plasticizers. Several other processes are also at the pilot plant scale and some ionic liquids are used commercially as additive e.g. binders in paints.

\section{3}

\section{What is an lonic Liquid?}

The recognised definition of an ionic liquid is "an ionic material that is liquid below $100^{\circ} \mathrm{C}$ " but leaves the significant question as to what constitutes an ionic material. Some authors limit the definition to cations with discrete anions e.g. $\mathrm{BF}_{4}{ }^{-}$, $\mathrm{NO}_{3}{ }^{-}$. This definition excludes the original work on chloroaluminate systems and the considerable work on other eutectic systems and is therefore unsatisfactory. Systems with anionic species formed by complex equilibria are difficult to categorise as the relative amounts of ionic species depend strongly on the composition of the different components.

Ionic liquids have also been separated into first and second generation liquids [10]; where first generation liquids are those based on eutectics and second generation have discrete anions [17]. Others have sought to further divide the first generation liquids into separate types depending on the nature of the Lewis or Brønsted acid that complexes [18]. While there is some dispute whether eutectics with Brønsted acids constitute ionic liquids at all there are others who seek to widen the description of ionic liquids to include materials such as salt hydrates [19].

In general, ionic liquids form because the charge on the ions is delocalized and this gives rise to a reduction in lattice energy. The majority of ionic liquids are 
described by the equilibrium:

$$
\text { cation }+ \text { anion }+ \text { complexing agent } \leftrightarrow \text { cation }+ \text { complex anion }
$$

Potentially, complex cations could also be formed using species such as cryptands or crown ethers:

$$
\text { cation }+ \text { anion }+ \text { complexing agent } \leftrightarrow \text { complex cation }+ \text { anion }
$$

The confusion arises from the magnitude of the equilibrium constant. For discrete anions such as $\mathrm{BF}_{4}{ }^{-}$and even $\left(\left(\mathrm{CF}_{3} \mathrm{SO}_{2}\right)_{2} \mathrm{~N}\right)^{-}$the equilibrium lies clearly to the right of Eq. (1.1). For some eutectic-based liquids the equilibrium constant is also to the right e.g.

$$
\mathrm{Cat}^{+} \mathrm{Cl}^{-}+\mathrm{AlCl}_{3} \leftrightarrow \mathrm{Cat}^{+}+\mathrm{AlCl}_{4}^{-}
$$

But the addition of more Lewis acid produces other anionic species.

$$
\mathrm{Cat}^{+} \mathrm{Cl}^{-}+2 \mathrm{AlCl}_{3} \leftrightarrow \mathrm{Cat}^{+}+\mathrm{Al}_{2} \mathrm{Cl}_{7}^{-}
$$

The use of less Lewis acidic metals e.g. $\mathrm{ZnCl}_{2}$ or $\mathrm{SnCl}_{2}$ will lead to a small amount of $\mathrm{Cl}^{-}$. The species formed between the anion and the complexing agent becomes weaker when a Brønsted acid e.g. urea is used [18].

$$
\mathrm{Cat}^{+} \mathrm{Cl}^{-}+\text {urea } \leftrightarrow \mathrm{Cat}^{+}+\mathrm{Cl}^{-} \cdot \text { urea }
$$

Others have claimed that, in the extreme, water can act as a good Brønsted acid and, in the extreme, hydrate salts can act as ionic liquids [19].

$$
\mathrm{LiClO}_{4}+3.5 \mathrm{H}_{2} \mathrm{O} \leftrightarrow \mathrm{Li}^{+} \cdot x \mathrm{H}_{2} \mathrm{O}+\mathrm{ClO}_{4}^{-} \cdot \gamma \mathrm{H}_{2} \mathrm{O}
$$

Ionic liquids with discrete anions have a fixed anion structure but in the eutecticbased liquids at some composition point the Lewis or Brønsted acid will be in considerable excess and the system becomes a solution of salt in the acid. A similar scenario also exists with the incorporation of diluents or impurities and hence we need to define at what composition an ionic liquid is formed. Many ionic liquids with discrete anions are hydrophilic and the absorption of water is found sometimes to have a significant effect upon the viscosity and conductivity of the liquid [20-22]. Two recent approaches to overcome this difficulty have been to classify ionic liquids in terms of their charge mobility characteristics [23] and the correlation between the molar conductivity and fluidity of the liquids [24]. This latter approach is thought by some to be due to the validity of the Walden rule

$$
\Lambda \eta=\mathrm{constant}
$$


61 Why use Ionic Liquids for Electrodeposition?

in ionic liquids, where $\Lambda$ is the molar conductivity and $\eta$ is the viscosity. This is, however a misrepresentation of Eq. (1.7) which was found empirically and is only strictly valid for a specific ion at infinite dilution and constant temperature. The Walden rule is a useful tool for approximate classification of ionic liquids but it actually follows from the Nernst-Stokes-Einstein equation (See Chapters 2.3 and 11.3) [23]. Most importantly, deviations from the Walden rule do not necessarily show that a salt is not an ionic liquid but more usually occur where ionic species deviate from the model of centro-symmetric spherical ions with similar ionic radii. The Walden rule can, however, be used to give evidence of different charge transfer mechanisms e.g. a Grotthus mechanism for protonic ionic liquids [24].

In this book a broad-church of ionic liquids will be assumed, encompassing all of the above types because, in the discipline of electrodeposition, it is the resultant deposit that is important rather than the means. As will be seen later there is also a very fine line between a concentrated electrolyte solution and an ionic liquid containing diluents.

1.4

Technological Potential of Ionic Liquids

A series of transition- and main group-metal-containing ionic liquids have been formulated and the feasibility of achieving electrodeposition has been demonstrated for the majority of these metals, Figure 1.1 shows the elements in the periodic table that have been deposited using ionic liquids. Details of these systems are given in the subsequent chapters and concise summaries exists in recently published reviews $[18,25,26]$.

It must be stressed that while the deposition of a wide range of metals has been demonstrated from a number of ionic liquids the practical aspects of controlling deposit morphology have not been significantly addressed due to the complex nature of the process parameters that still need to be understood. Despite the lack of reliable models to describe mass transport and material growth in ionic liquids, there are tantalizing advantages that ionic liquid solvents have over aqueous baths that make the understanding of their properties vitally important. Some of these advantages include:

- Electroplating of a range of metals impossible to deposit in water due to hydrolysis e.g. Al, Ti, Ta, Nb, Mo, W. As an example, the deposition of Al by electrolysis in a low-temperature process has long been a highly desirable goal, with many potential applications in aerospace for anti-friction properties, as well as replacing $\mathrm{Cr}$ in decorative coatings. The deposition of $\mathrm{Ti}, \mathrm{Ta}, \mathrm{Nb}, \mathrm{Mo}$, W will open important opportunities in various industries, because of their specific properties (heat, corrosion, abrasion resistance, low or high density etc.).

- Direct electroplating of metals on water-sensitive substrate materials such as Al, $\mathrm{Mg}$ and light alloys with good adherence should be possible using ionic liquids.

- There is potential for quality coatings to be obtained with ionic liquids rather than with water. Currently available metallic coatings suffer from hydrogen 


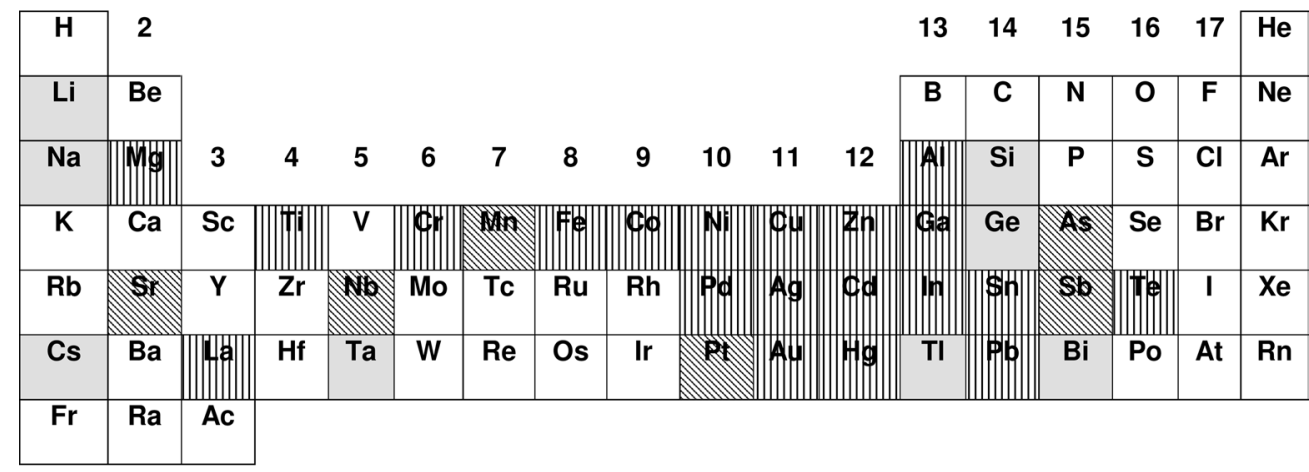

\begin{tabular}{|c|c|c|c|c|c|c|c|c|c|c|c|c|c|}
\hline $\mathrm{Ce}$ & $\mathrm{Pr}$ & Wo & Pm & $\mathrm{Sm}$ & Eu & Gd & Tb & 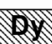 & Ho & $\mathrm{Er}$ & Tm & $\mathbf{Y b}$ & Lu \\
\hline Th & $\mathrm{Pa}$ & $\psi$ & $\mathrm{Np}$ & $\mathbf{P u}$ & Am & $\mathrm{Cm}$ & Bk & Cf & Es & $\mathrm{Fm}$ & Md & No & $\mathbf{L r}$ \\
\hline
\end{tabular}

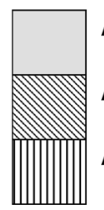

As Metal

As Alloy

As Metal and Alloy

Fig. 1.1 Summary of the elements deposited as single metals or alloys.

embrittlement; a major problem caused by gaseous hydrogen produced during water electrolysis. During electroplating with ionic liquids, negligible hydrogen is produced, and coatings will have the better mechanical properties.

- Metal ion electrodeposition potentials are much closer together in ionic liquids compared with water, enabling easier preparation of alloys and the possibility of a much wider range of possible electroplated alloys, which are difficult or impossible in water.

- Ionic liquids complex metals and therefore offer the possibility to develop novel electroless plating baths for coating polymers (e.g. in electronics) without the need for the toxic and problematic organic complexants used in water.

- Although the cost of ionic liquids will be greater than aqueous electrolytes, high conductivity and better efficiency will provide significant energy savings compared with water, and capital costs will be much lower than the alternative techniques PVD and CVD.

- When used in electropolishing and electropickling processes, strongly acidic aqueous electrolytes create large quantities of metal-laden, corrosive effluent solution, whereas in ionic liquid electrolytes the metals will precipitate and be readily separated and recycled. 
8 ( 1 Why use lonic Liquids for Electrodeposition?

- The replacement of many hazardous and toxic materials currently used in water, e.g. toxic form of chromium(VI), cyanide, highly corrosive and caustic electrolytes, would save about $10 \%$ of the current treatment costs.

- Nanocomposite coatings - nanoparticles giving improved properties compared to microparticles e.g. thermal and electrical conductivity, transparency, uniformity, low friction.

- An increased range of metal coatings on polymers is accessible by electroless plating using ionic liquids containing reducing agents

In the longer term, specialist, ionic liquids will enable technically complex highadded-value products to be introduced, e.g. semiconductor coatings, special magnetic alloys, nanoparticle composite coatings with special erosion/corrosion properties, metal foams for energy storage activated surfaces for self-sterilization purposes (e.g. through photo-catalysis), etc.

Also, metals have significantly different reduction potentials in ionic liquid solutions compared to water. For example the difference in reduction potential between $\mathrm{Cr}$ and $\mathrm{Pt}$ in ionic liquids may be as little as $100 \mathrm{mV}$ whereas in aqueous solutions it is in excess of $2 \mathrm{~V}$. One consequence of this characteristic is that alloy coatings may be prepared more readily and that it should be possible to develop many novel alloy coatings.

A fundamental advantage of using ionic liquid electrolytes in electroplating is that, since these are non-aqueous solutions, there is negligible hydrogen evolution during electroplating and the coatings possess the much superior mechanical properties of the pure metal. Hence essentially crack-free, more corrosion-resistant deposits are possible. This may allow thinner deposits to be used, thus reducing overall material and power consumption still further.

The electrodeposition of metals from ionic liquids is a novel method for the production of nanocrystalline metals and alloys, because the grain size can be adjusted by varying the electrochemical parameters such as over-potential, current density, pulse parameters, bath composition and temperature and the liquids themselves. Recently, for the first time, nanocrystalline electrodeposition of Al, Fe and Al-Mn alloy has been demonstrated.

The properties of the new electrolyte media could also provide much higher health and safety standards for employees in the workplace, i.e. elimination of hazardous vapors, elimination of highly corrosive acidic/alkaline solutions and substantially reduced use of toxic chemicals. These issues are dealt with later in the book. Current aqueous processing systems have a strongly negative impact on the environment (risk of groundwater contamination, soil pollution), which obliges the treatment of wastewater and the dumping of the ultimate waste in landfill. The metal finishing industry in general estimates that at least $15 \%$ of turnover is related to the cost of treatment for environmental protection. Legislation within the framework of sustainable development is increasingly stringent (e.g. European Commission directive 96/61/EC concerning "integrated pollution prevention and control”). Thus, industries using metal finishing processes must search for new techniques to achieve these environmental goals. In addition to the growing costs 
and negative effects on competitiveness, it is a question of survival in the years to come. The technology developed by this project is generic to most metal-plating systems and, as such, should represent a significant advancement for the environmental sustainability of the metal finishing and electronics manufacturing sectors.

Ionic liquids are based on large non-centrosymmetric organic cations with complex anions, which are liquid at room temperature. The range of new ionic liquids has insignificant vapor pressure (thus odorless), some are non-toxic (and even completely biodegradable) and most are highly conductive compared with organic electrolyte solutions. This statement has to be tempered, naturally, because compared to the current state of the art, i.e. concentrated inorganic acids, the conductivities are at best 10 to 100 times lower. An advantage might be that ionic liquids can be operated at temperatures above $100^{\circ} \mathrm{C}$ where ionic conductivities of up to $0.2 \Omega^{-1} \mathrm{~cm}^{-1}$ are achievable. The ongoing development of ionic liquids might lead to even better conducting liquids.

There are, however numerous risk elements in the development of ionic liquids:

- Coatings must achieve quality standards and a large amount of process development is required.

- A life cycle analysis (LCA) and an environmental impact study have not been completed for any of this technology.

- Issues of scale-up and integration design of generic prototype systems have not been addressed systematically.

- Some applications are at a fundamental research stage with associated higher risk, i.e. electroless coating, semiconductors, anodising, nanocomposite coatings.

- Process economics are expected to be favorable for high-added-value products, but there are likely to be applications where economics are less favorable.

- For improved existing products, customer acceptance is likely to be a significant factor, i.e. reluctance to change product specifications.

The potential impact is extremely broad and fundamental in nature, because the research will explore a totally innovative approach to metal finishing technology, which has never been exploited previously. The use of this completely different type of solvent/electrolyte system, entirely changes the normal behavior of metal finishing processes seen in traditional aqueous electrolytes and an extensive range of entirely new processes and products can be expected.

The following chapters discuss the history, development and physical properties of low-temperature ionic substances but in this section it is useful to discuss the differences that arise in changing from a molecular to an ionic environment and the implications that this will have for electrodeposition processes occurring at an electrode surfaces.

There are several physical plating parameters that are different in an ionic liquid from those in an aqueous solution.

Temperature: Ionic liquids have wide liquid regions, typically in the range -50 to $250^{\circ} \mathrm{C}$ which allow more thermodynamic control than is possible in aqueous solutions. This may have potential benefits for the development of new alloys. 
Diluents: Ionic liquids can be diluted with a range of organic and aqueous solvents and these significantly affect conductivity, viscosity and metal speciation. The effects have not as yet been characterized and a significant amount of fundamental data still needs to be obtained. A significant amount of work has been carried out on the chloroaluminate-based ionic liquids, although their use in other ionic liquids has been generally ignored.

Cation: Cationic structure and size will affect the viscosity and conductivity of the liquid and hence will control mass transport of metal ions to the electrode surface. They will also be adsorbed at the electrode surface at the deposition potential and hence the structure of the double layer is dominated by cations. Some studies have shown that changing the cationic component of the ionic liquid changes the structure of deposits from microcrystalline to nanocrystalline [27]. While these changes are undeniable more studies need to be carried out to confirm that it is a double layer effect. If this is in fact the case then the potential exists to use the cationic component in the liquid as a built-in brightener.

Double Layer Structure: Surprisingly few studies have been carried out into the double layer structure of ionic liquids. This is partially due to experimental difficulties but also to interpretation of the resulting impedance spectra. What is clearly evident, however, is that the double layer in an ionic liquid cannot be described by applying the models used for aqueous solutions [28-30]. A study using imidazolium bistriflamide, $\left(\mathrm{F}_{3} \mathrm{CSO}_{2}\right)_{2} \mathrm{~N}^{-}$and $\mathrm{BF}_{4}{ }^{-}$salts suggested that a model of alternating anion and cation layers may be applicable to the data [29, 30]. Baldelli $[31,32]$ concluded that the double layer is one ion layer thick using sum frequency generation spectroscopy and electrochemistry to probe the electric field at the ionic liquid/electrode interface. The double layer capacitance in an ionic liquid is considerably smaller than in an aqueous solution and less than that predicted by having a perfect Helmholtz layer at the interface, which could result from the presence of ion pairs at the electrode surface at all potentials. Most likely the double layer structure is also influenced by cation/anion interaction.

While the structure at the electrode/ionic liquid interface is uncertain it is clear that in the absence of neutral molecules the concentration of anions and cations at the interface will be potential dependent. The main difference between aqueous solutions and ionic liquids is the size of the ions. The ionic radii of most metal ions are in the range $1-2 \AA$ whereas for most ions of an ionic liquid they are more typically $3-5 \AA$. This means that in an ionic liquid the electrode will be coated with a layer of ions at least $6-7 \AA$ thick. To dissolve in an ionic liquid most metal species are anionic and hence the concentration of metal ions close to the electrode surface will be potential dependent. The more negative the applied potential the smaller the concentration of anions. This means that reactive metals such as $\mathrm{Al}, \mathrm{Ta}, \mathrm{Ti}$ and $\mathrm{W}$ will be difficult to deposit as the effective concentration of metal might be too low to nucleate. It is proposed, as one explanation, that this is the reason that aluminum cannot be electrodeposited from Lewis basic chloroaluminate ionic liquids. More reactive metals such as lithium can however be deposited using ionic liquids because they are cationic and therefore 
present at high concentrations close to the electrode surface at large negative overpotentials. The strategy to electrodeposit reactive metals must therefore be to either make cationic metal complexes or to work with metal salts at high concentrations.

Anode material: In aqueous solutions the anodic processes are either breakdown of the electrolyte solution (with oxygen evolution at an inert anode being favored) or the use of soluble anodes. The use of soluble anodes is limited by the passivation of many metals in aqueous solutions. In ionic liquids, however, the first option is not viable due to the cost and the nature of the anodic breakdown products. New strategies will therefore have to be developed to use soluble anodes where possible or add a sacrificial species that is oxidized to give a benign gaseous product. Preliminary data have shown that for some metals the anodic dissolution process is rate limiting and this affects the current distribution around the cathode and the current density that can be applied.

Electrolytes: The above issue of double layer structure is important to the mechanism of nucleation and growth in ionic liquids, it may therefore be possible to control the structure at the electrode/solution interface by addition of an inert electrolyte. In this respect most Group 1 metals are soluble in most ionic liquids, although it is only generally lithium salts that exhibit high solubility. In ionic liquids with discrete anions the presence of Group 1 metal ions can be detrimental to the deposition of reactive metals such as $\mathrm{Al}$ and Ta where they have been shown to be co-deposited despite their presence in trace concentrations.

Brighteners: Brighteners are added to most aqueous electroplating solutions and work by either complexing the metal ions and decreasing the rate of nucleation or by acting as an interfacial adsorbate, blocking nucleation and hindering growth. Aqueous brighteners have not been studied in depth in ionic liquids and it is doubtful that they will function in the same way as they do in water because of the difference in double layer structure and mass transport. In unpublished work we have surveyed the use of aqueous brightener compounds and applied them to the deposition of zinc and chromium from Type 2 or Type 3 eutectics (see also Chapter 2). None of these were found to be effective in ionic liquids.

A small amount of work has been carried out into brighteners that complex the metal ions in solution (see Chapter 11.3) but again no systematic studies have been carried out. Brighteners which rely on electrostatic or hydrophobic interactions may function in ionic liquids but their efficacy is likely to be surface and cation/anion specific. To date all systems that have produced bright metallic finishes have been found to have a nanocrystalline structure which may be due to a progressive nucleation mechanism. This is currently under investigation and if confirmed it will help significantly with the design of future brightener systems.

As with other solutes in ionic liquids, the general rule of like dissolving like is applicable i.e. ionic species will generally be soluble as will species capable of interacting with the anion. Aromatic species tend to exhibit poor solubility in ionic liquids consisting of aliphatic cations and vice versa. 
1 Why use lonic Liquids for Electrodeposition?

1.5

Concluding Remarks

What is clear from this introduction is that the journey into the area of metal deposition from ionic liquids has tantalizing benefits. It is also clear that we have only just begun to scratch the surface of this topic. Our models for the physical properties of these novel fluids are only in an early state of development and considerably more work is required to understand issues such as mass transport, speciation and double layer structure. Nucleation and growth mechanisms in ionic liquids will be considerably more complex than in their aqueous counterparts but the potential to adjust mass transport, composition and speciation independently for numerous metal ions opens the opportunity to deposit new metals, alloys and composite materials which have hitherto been outside the grasp of electroplaters.

\section{References}

1 (2000) Modern Electroplating, 4th edn (eds M. Schlesinger and M. Paunovic), John Wiley \& Sons, Inc., New York.

2 Peled, E. and Gileadi, E. (1976) J. Electrochem. Soc., 123, 15-19.

3 Simanavicius, L. (1990) Chemija, 3, 3-30.

4 Izutsu, K. (2002) Electrochemistry in Non-aqueous Solutions, Wiley-VCH, Verlag GmbH.

5 Kruesi, W.H. and Fray, D.J. (1993) Metall. Trans. B., 24, 605.

6 Fray, D.J. and Chen, G.Z. (2004) Mater. Sci. Technol., 20, 295.

7 Grjotheim, K., Krohn, C., Malinovsky, M., Matiasovsky, K., and Thonstad, J. (1982) Aluminum Electrolysis, 2nd edn, Aluminium-Verlag, Dusseldorf.

8 Lantelme, F., Alexopoulos, H., Chemla, M., and Haas, O. (1988) Electrochim. Acta, 33, 761.

9 Wasserscheid, P. and Welton, T. (2003) Ionic Liquids in Synthesis, Wiley-VCH, Verlag GmbH.

10 Welton, T. (1999) Chem. Rev., 99, 2071.

11 Wasserscheid, P. and Keim, W. (2000) Angew. Chem. Int. Ed., 39, 3772.

12 Earle, M.J. and Seddon, K.R. (2000) Pure Appl. Chem., 72, 1391.

13 Zhang, S. and Conrad Zhang, Z. (2002) Green Chemistry, 4, 376.

14 Whitehead, J.A., Lawrence, G.A., and McCluskey, A. (2004) Green Chem., 6, 313.
15 Maase, M. (2005) in Multiphase Homogeneous Catalysis (eds B. Cornils et al.), Wiley-VCH, Weinheim, Germany, p. 560.

16 Chauvin, Y., Olivier, H., Wyrvalski, C.N., Simon, L.C., de Souza, R., and Dupont, J. (1997) J. Catal., 165, 275.

17 Chiappe, C. and Pieraccini, D. (2005) J. Phys. Org. Chem., 18, 275-297.

18 Abbott, A.P. and McKenzie, K.J. (2006) Phys. Chem. Chem. Phys., 8, 4265-4279.

$19 \mathrm{Xu}, \mathrm{W}$. and Angell, C.A. (2003) Science, 302, 422.

20 Billard, I., Mekki, S., Gaillard, C., Hesemann, P., Moutiers, G., Mariet, C., Labet, A., and Buenzli, J.G. (2004) Eur. J. Inorg. Chem., 6, 1190-1197.

21 Jarosik, A., Krajewski, S.R., Lewandowski, A., and Radzimski, P. (2006) J. Mol. Liq., 123, 43-50.

22 Widegren, J.A., Saurer, E.M., Marsh, K.N., and Magee, J.W. (2005) J. Chem. Thermodyn., 37, 569-575.

23 Abbott, A.P., Harris, R.C., and Ryder, K.S. (2007) J. Phys. Chem. B, 111, 4910-4914.

24 Yoshizawa, M., Xu, W., and Angell, C.A. (2003) J. Am. Chem. Soc., 125, 15411.

25 Endres, F. and Zein El Abedin, S. (2006) Phys. Chem. Chem. Phys., 8, 2101.

26 Zein El-Abedin, S. and Endres, F. (2002) Phys. Chem. Chem. Phys., 4, 1640. 
c01 (JWBG008-Endres) December 25, 2007 13:13 Char Count=

\section{References $\mid 13$}

27 Moustafa, E.M., Zein El Abedin, S., Shkurankov, A., Zschippang, E., Saad, A.Y., Bund, A., and Endres, F. (2007) J. Phys. Chem. B, 111, 4693.

28 Gale, R.J. and Osteryoung, R.A. (1980) Electrochim. Acta, 25, 1527.

29 Nanjundiah, C., McDevitt, S.F., and Koch, V.R. (1997) J. Electrochem. Soc., 144, 3392.
30 Nanjundiah, C., Goldman, J.L., McDevitt, S.F., and Koch, V.R. (1997) Proc. Electrochem. Soc., 96-25, 301.

31 Baldelli, S. (2005) J. Phys. Chem. B, 27, 109.

32 Rivera-Rubero, S. and Baldelli, S. (2004) J. Phys. Chem. B, 108, 15133. 
